\title{
Genomic diversity and ecology of human- associated Akkermansia species in the gut microbiome revealed by extensive metagenomic assembly
}

Nicolai Karcher ${ }^{1 \dagger}$, Eleonora Nigro ${ }^{2 \dagger}$, Michal Punčocháŕ ${ }^{1}$, Aitor Blanco-Míguez ${ }^{1}$, Matteo Ciciani ${ }^{1}$, Paolo Manghi ${ }^{1}$, Moreno Zolfo', Fabio Cumbo', Serena Manara', Davide Golzato', Anna Cereseto', Manimozhiyan Arumugam², Thi Phuong Nam Bui ${ }^{3}$, Hanne L. P. Tytgat ${ }^{3,4}$, Mireia Valles-Colomer ${ }^{1 \dagger}$, Willem M. de Vos ${ }^{3,5+}$ and Nicola Segata ${ }^{1,6^{*}+}$ (D)

\author{
* Correspondence: nicola.segata@ \\ unitn.it \\ ${ }^{\dagger}$ Nicolai Karcher and Eleonora Nigro \\ contributed equally to this work. \\ ${ }^{\dagger}$ Mireia Valles-Colomer, Willem M. \\ de Vos, and Nicola Segata are co- \\ senior authors. \\ ${ }^{1}$ Department $\mathrm{CIBIO}$, University of \\ Trento, Trento, Italy \\ ${ }^{6} \mathrm{IEO}$, European Institute of \\ Oncology IRCCS, Milan, Italy \\ Full list of author information is \\ available at the end of the article
}

\begin{abstract}
Background: Akkermansia muciniphila is a human gut microbe with a key role in the physiology of the intestinal mucus layer and reported associations with decreased body mass and increased gut barrier function and health. Despite its biomedical relevance, the genomic diversity of $A$. muciniphila remains understudied and that of closely related species, except for $A$. glycaniphila, unexplored.

Results: We present a large-scale population genomics analysis of the Akkermansia genus using 188 isolate genomes and 2226 genomes assembled from 18,600 metagenomes from humans and other animals. While we do not detect $A$. glycaniphila, the Akkermansia strains in the human gut can be grouped into five distinct candidate species, including A. muciniphila, that show remarkable wholegenome divergence despite surprisingly similar $16 \mathrm{~S}$ rRNA gene sequences. These candidate species are likely human-specific, as they are detected in mice and nonhuman primates almost exclusively when kept in captivity. In humans, Akkermansia candidate species display ecological co-exclusion, diversified functional capabilities, and distinct patterns of associations with host body mass. Analysis of CRISPR-Cas loci reveals new variants and spacers targeting newly discovered putative bacteriophages. Remarkably, we observe an increased relative abundance of Akkermansia when cognate predicted bacteriophages are present, suggesting ecological interactions. A. muciniphila further exhibits subspecies-level genetic stratification with associated functional differences such as a putative exo/ lipopolysaccharide operon.

Conclusions: We uncover a large phylogenetic and functional diversity of the Akkermansia genus in humans. This variability should be considered in the ongoing experimental and metagenomic efforts to characterize the health-associated properties of A. muciniphila and related bacteria.
\end{abstract}




\section{Introduction}

The human body is home to several distinct microbiomes which represent functionally and phylogenetically diverse microbial ecosystems that are key for human health [1-3]. A frequent and abundant inhabitant of the gut microbiome is Akkermansia muciniphila, a Gram-negative, non-motile anaerobic bacterium specialized in the degradation of mucin [4]. A. muciniphila can utilize mucin as its sole carbon and nitrogen source [4]; thus, growth in its natural habitat is not directly dependent on the influx of dietary compounds. A. muciniphila continues to attract attention due to its association with host health: the relative abundance of $A$. muciniphila is inversely correlated with obesity in humans $[5,6]$, and it was shown to alleviate insulin resistance and obesity while increasing gut barrier function in a mouse model of diet-induced obesity [7]. Its potential as a next-generation probiotic in the battle against metabolic disorders was confirmed in a first intervention trial targeting humans with metabolic syndrome and obesity [8].

The human microbiome hosts a vast bacterial diversity at the level of distinct strains belonging to the same species (i.e., conspecific strains) [3, 9-13]. The genomic variation of conspecific strains often exceeds $3 \%$ nucleotide variation in the core genes, and when comparing pairs of conspecific strains, it is frequently observed that $25 \%$ of genes are present in only one of the two, causing each human microbiome to be unique at the strain level [3]. Importantly, this subspecies genomic variability translates into phenotypic variability, for example, in connection with host lifestyle [14-16] and at the immunological level $[17,18]$. However, experimental Akkermansia research still heavily relies on the type strain A. muciniphila $\mathrm{Muc}^{\mathrm{T}}$ (ATCC BAA-835) and on a few more genomes of newly isolated strains that became available recently [19-21]. Furthermore, only a single other species in the Akkermansia genus-A. glycaniphila $\left(\mathrm{Pyt}^{\mathrm{T}}\right.$, DSM 100705) - has so far been described and genomically characterized [22, 23]. There is thus the urgent need to expand our understanding of the genomic variation and (sub)species diversity of Akkermansia for improving both the interpretation of its functions and its potential use in biomedicine.

Recently, a large number of publicly available metagenomes of human-associated microbial communities have been mined to produce hundreds of thousands of metagenome-assembled genomes (MAGs) [3, 24-26] and methods to profile and investigate strains directly in metagenomes have become increasingly effective $[9,10,27-$ 29]. While these tools offer the opportunity to characterize population genomics of important but poorly characterized human-associated bacteria, only a few species have been investigated so far at high genomic resolution and global scale [30-33].

Here, we present a comprehensive genomic characterization of Akkermansia muciniphila and closely related Akkermansia spp., using a total of 2226 MAGs belonging to the Akkermansia genus, 188 publicly available isolate genomes, and 6 newly sequenced isolate genomes. The Akkermansia MAGs were obtained by expanding our recent catalog of human-associated MAGs [3] with 166,518 additional MAGs from 45 different datasets comprising samples also from mice and non-human primates for an integrated catalog of 321,241 MAGs (see the "Methods" section). Next to the species-level clade with the A. muciniphila type strain, we show the existence of four other Akkermansia candidate species that colonize the human gut. These five candidate species display strong co-exclusion within a given host, are phylogenetically stratified at the subspecies 
level, and are at the same time widely distributed across hosts, age, and geography. Comparison of candidate species shows differential association with BMI in humans and vitamin B12 synthesis capabilities. We also analyzed the genomic organization of CRISPR-Cas loci (providing adaptive immunity against foreign DNA [34]) in Akkermansia candidate species and found these to differ in their locus architecture and spacer numbers. We furthermore identified de novo assembled putative bacteriophages with spacer hits from Akkermansia candidate species and found that viral detectability correlates strongly with the relative abundance of cognate Akkermansia candidate species, suggesting an intimate ecological interplay. These, and other genomic analyses in this work, provide a solid basis for future mechanistic explorations and biomedical applications of Akkermansia.

\section{Results and discussion}

\section{A large-scale metagenomics-based analysis of Akkermansia candidate species}

In order to study the diversity of Akkermansia species in the human microbiome, we collected all genomes available from isolate sequencing as well as MAGs from large collections of metagenomic datasets and unified them into a single genomic resource. Specifically, we gathered and quality controlled 119 isolate genomes from NCBI that were taxonomically annotated as A. muciniphila, as well as 69 labeled as Akkermansia sp. [4, 5, 19, 22, 35-41]. We further obtained 2226 MAGs taxonomically annotated to the genus Akkermansia from a total of 18,600 shotgun metagenomes (see the "Methods" section) sampled from multiple hosts including humans, non-human primates, and mice. Only high-quality MAGs-defined as those with at least $90 \%$ estimated genomic completeness and at most 5\% estimated genomic contamination [42] - were included in the analysis. We further enhanced our genome set with 6 isolate genomes [43]. The integrated Akkermansia genome resource we consider for downstream analysis thus consists of a total of 2420 genomes (Additional file 1: Table S1).

\section{Multiple under-characterized Akkermansia candidate species are present in the human microbiome}

We reconstructed the phylogeny of all genomes in our set using the 400 universal marker genes adopted in PhyloPhlAn 3 [44-46] (Fig. 1A, the "Methods" section) including Verrucomicrobium spinosum as an outgroup [4]. This revealed the presence of several well-defined monophyletic clades (Fig. 1A). In addition to the previously described A. glycaniphila species [22], these clades-following the validated species-level genome bins (SGBs) approach based on whole-genome genetic distances [3] (see the "Methods" section) - delineate candidate species (Fig. 1B). The candidate species are genetically distinct, with inter-candidate species genome-wide average estimated nucleotide identities generally below $90 \%$ (except between a single pair of candidate species, Fig. 1B). We confirmed those results using genome similarity estimates obtained using PhyloPhlAn 3 markers (Additional file 2: Figure S1). One of the five delineated candidate species (henceforth "A. muciniphila") includes the type strain of A. muciniphila $\left(\mathrm{Muc}^{\mathrm{T}}\right)$ [4] as well as 108 isolate genomes. The remaining four candidate species (SGB9223, SGB9224, SGB9227, and SGB9228) comprise not only MAGs but also isolate genomes that were, however, taxonomically described as A. muciniphila or 

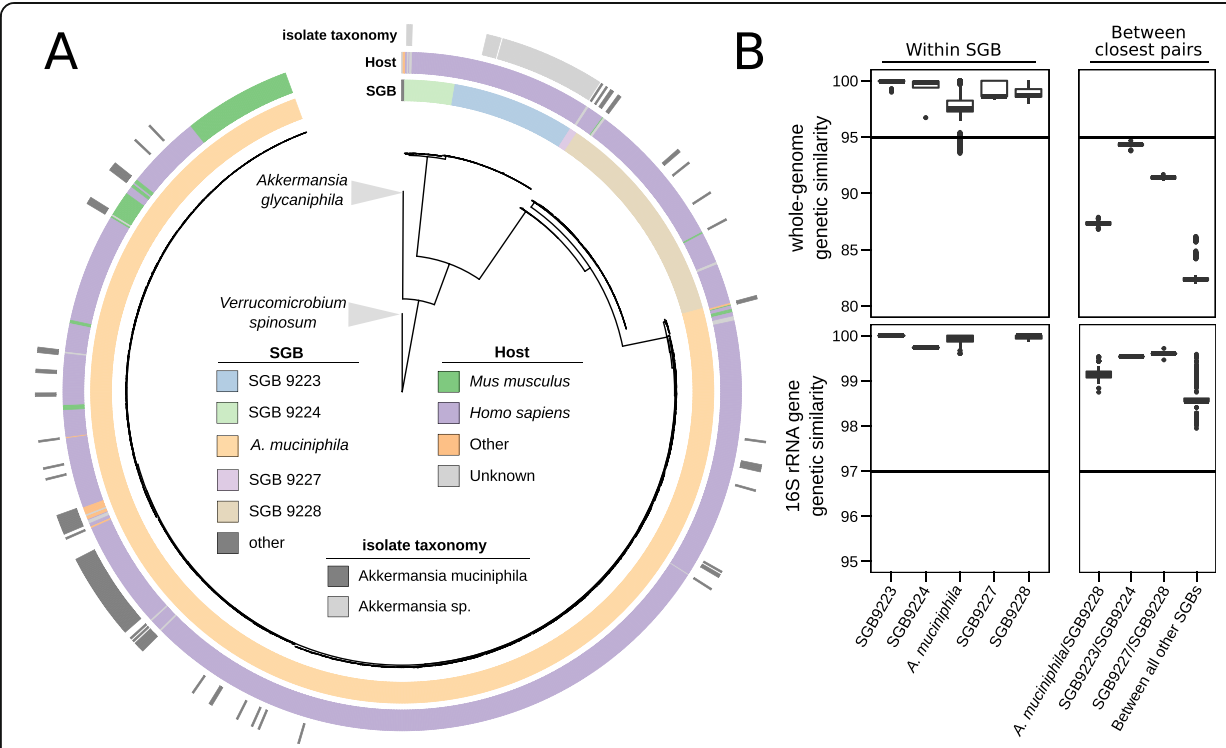

Fig. 1 The Akkermansia genus comprises four additional candidate species phylogenetically rooted between the already characterized A. glycaniphila and A. muciniphila. A Whole-genome phylogeny of the 2420 metagenome-assembled genomes (MAGs) reconstructed here and the genomes from isolate sequencing available in NCBI taxonomically annotated as A. muciniphila or Akkermansia spp. The phylogenetic tree is rooted using Verrucomicrobium spinosum as an outgroup and was built using PhyloPhIAn 3 [46] with 400 universal markers (see the "Methods" section). SGB, species-level genome bin (see the "Methods" section). B Within- and between-clade whole-genome average estimated nucleotide identity (fastANI [47], top panels) and full-length $16 \mathrm{~S}$ sequence distances (bottom panels) among Akkermansia SGBs provide evidence that these are candidate species

Akkermansia spp. in NCBI. Cultivated members of the candidate species were retrieved not only from humans, but also from mice, non-human primates, and-very rarelyother mammals, such as elephants, horses, and reindeers [20]. A. glycaniphila was originally isolated from a python [22], and we did not uncover new diversity for this species in the available datasets, suggesting that A. glycaniphila is not found in mammals. A reason for the taxonomic assignment of cultivated strains to the A. muciniphila species and the generally underestimated diversity of the genus is probably the surprisingly high similarity displayed by $16 \mathrm{~S}$ rRNA gene sequences of these strains, with $16 \mathrm{~S}$ rRNA gene sequences of strains in different candidate species never diverging by more than 2\% (Fig. 1B, the "Methods" section). Taken together, these data show that a total of five Akkermansia candidate species exist in the human, mouse, and non-human primate gut microbiomes, four of them remaining under-investigated and uncharacterized.

\section{Akkermansia candidate species are enriched in humans and co-exclude within a host}

We next set out to assess host specificity, co-abundance patterns, and metadata associations of the Akkermansia candidate species. To this end, we first developed a markerbased method with increased sensitivity compared to metagenomic assembly to detect the presence and relative abundance of Akkermansia candidate species in metagenomes. In brief, this was done by (1) identifying genes that were core to each of the Akkermansia candidate species and at the same time never detected in other Akkermansia or non-Akkermansia species-level groups (marker genes) and (2) using these 
marker genes as targets for read-mapping inside MetaPhlAn $3.0[48,49]$ to estimate their coverage and relative abundance (see the "Methods" section). By profiling the 13, 237 metagenomic samples from 98 publicly available datasets with sufficient metadata (Additional file 1: Table S2), we found that Akkermansia candidate species differed strongly in their prevalence across hosts. A. muciniphila is by far the most prevalent candidate species across all hosts, being detected in 34\% of adult humans and reaching a maximum prevalence of $54 \%$ in laboratory-held mice (Fig. 2A). The other candidate species were detected at lower prevalence $(<25 \%)$ across all hosts (Fig. 2A). Interestingly, Akkermansia candidate species were found to be much more often present in captive animals than in free-living ones: while laboratory mice and mice humanized via microbiome transplantation are fairly likely to host A. muciniphila, SGB9224, or

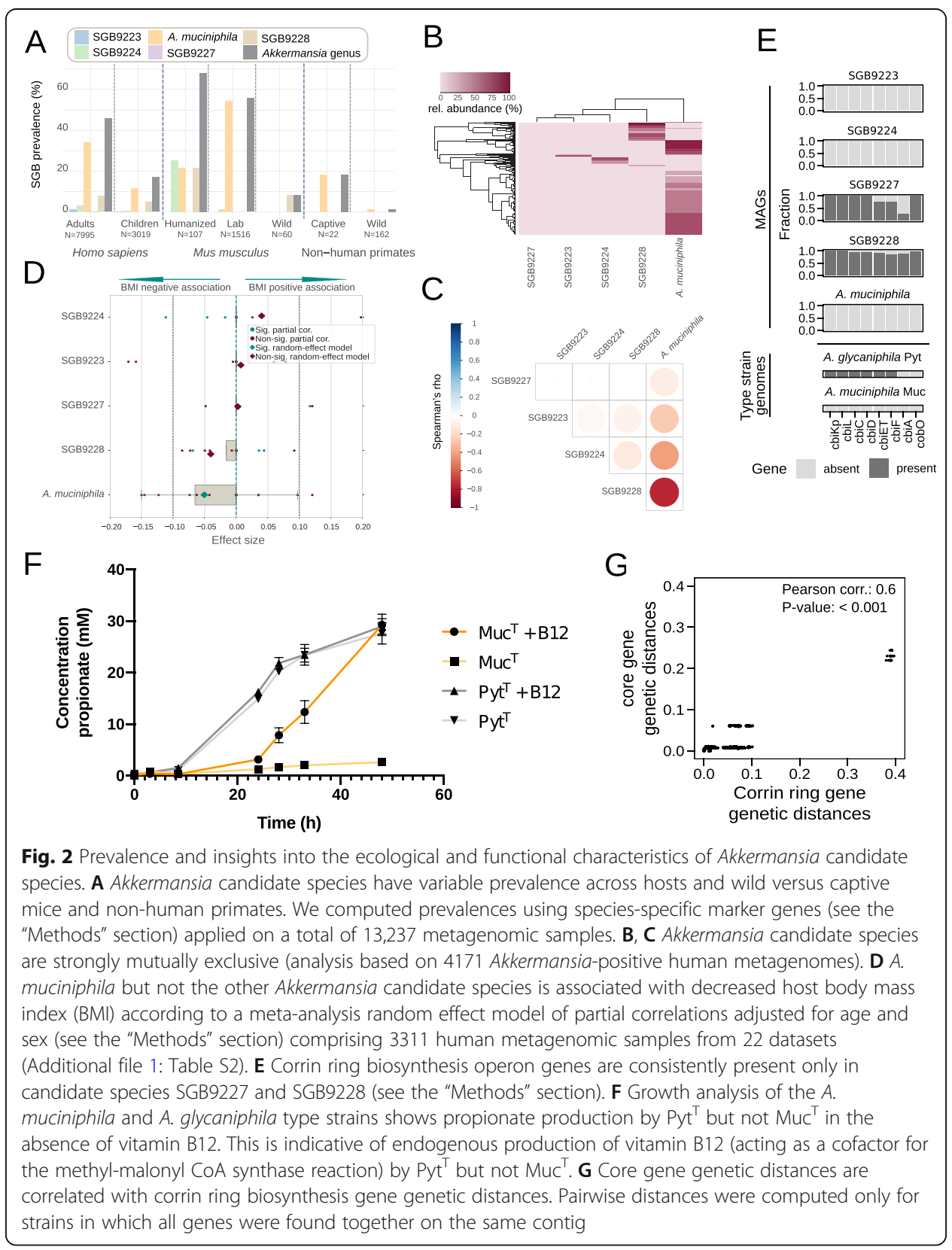


SGB9228 (up to $54 \%$ prevalence), in wild mice, solely SGB9228 was detected in only 5 mice from a single study (out of 60 mice from 3 different studies being assessed; 8\% prevalence). Similarly, merely two out of 162 samples from wild non-human primates tested positive for any Akkermansia candidate species (1.2\% prevalence). Despite potential biases due to uneven sampling and effects of diet, these data suggest a marked specificity of Akkermansia candidate species for the human gut (with the exception of $A$. glycaniphila), and while strains from these candidate species can colonize mice and non-human primates, such colonization appears to be predominantly a consequence of man-made environments, suggesting colonization from care-taking humans as a plausible mechanism.

While Akkermansia candidate species are found in almost half of all human samples, the presence of one is strongly anti-correlated with the others (Fig. 2B, C): it is extremely rare to detect more than one candidate species present in the same host, with only 46 instances of two co-occurring candidate species in human metagenomes out of 4171 cases in which at least one was present (corresponding to a co-occurrence rate of $\sim 1 \%$ ), and no instance of more than two co-occurring candidate species. These five closely related candidate species thus show a mutual exclusion pattern suggestive of complex and possibly host-mediated ecological interactions that remain to be explored.

\section{A. muciniphila but not the other candidate species is associated with lower BMI}

The presence and abundance of $A$. muciniphila in the gut microbiome has been negatively associated with body mass index in previous studies based on 16S rRNA gene sequencing $[50,51]$, and the link was shown to be causal in both mice and humans by supplementation with cells of $A$. muciniphila $\mathrm{Muc}^{\mathrm{T}}[7,8]$. Because of the limitations of 16S rRNA gene amplicon sequencing in distinguishing Akkermansia candidate species (Fig. 1B), we performed an analysis on the association between relative abundances of individual Akkermansia candidate species and BMI. We considered 3311 samples in 22 different metagenomic datasets from five continents and adjusted for age and sex in a random effect model meta-analysis (Additional file 1: Table S3). Interestingly, only the relative abundances of $A$. muciniphila were found to be significantly negatively associated with BMI, while associations of other candidate species were not statistically significant (Fig. 2D), suggesting that A. muciniphila should be regarded as the primary candidate for microbiota-based therapeutic interventions aimed at improving host metabolic health (as a recent proof-of-concept trial also reported [8]).

We next tested whether available host characteristics other than BMI were associated with Akkermansia candidate species relative abundances and also examined whether genetic stratification by host parameters could be detected within candidate species. At the candidate species-level, no association with age was detected, while sex (as selfdeclared by the individuals) was associated with the relative abundance of A. muciniphila (after adjusting for age and BMI), with women harboring comparatively higher relative abundances $(P$-value $=4.8 \mathrm{e}-05)$, as observed elsewhere [52]. To test for associations of candidate species with host metadata at the level of their internal phylogenomic structure, we subsequently computed PERMANOVA statistics for all combinations of single candidate species and host, age, geography, and Westernization status. While some significant associations were identified (especially for A. muciniphila 
and SGB9228), the largest effect size among the significant $(P$-value $<0.05)$ tests was a PERMANOVA $\mathrm{R}^{2}$ of 0.10 for SGB9228 with continent (Additional file 2: Figures S2, S3, S4), suggesting that no strong associations of strain-level structure with host metadata are detectable.

With Akkermansia supplementation becoming available $[8,53]$, it is relevant to verify that such interventions are not potentially causing microbial anti-drug resistances to spread in the human gut. To this end, we first screened all Akkermansia genomes for antibiotic resistance genes using the Comprehensive Antibiotic Resistance Database (CARD) [54]. Overall, we found only 4 genes known to be involved in antibiotic resistance present in more than $1 \%$ of all genomes. Among those, only adeF (encoding a membrane protein of a drug efflux complex) is consistently found in most genomes (prevalence of 81\% over all genomes), but still never present in SGB9223, SGB9224, nor SGB9227 (Additional file 2: Figure S5). In addition to these well-cataloged resistance genes, a recent study reported the presence of 8 genes (including 3 antibiotic resistance genes) in A. muciniphila strain GP36 derived from the broad-range plasmid RSF1010 that is found in many gram-negative bacteria including E. coli [19]. We queried all genomes for the presence of this plasmid-derived sequence and found 55 genomes $(2.2 \%$ overall prevalence) in which we could detect at least $50 \%$ of the sequence of RSF1010 at 70\% average sequence identity or higher. A total of 49 of the 55 instances were found in A. muciniphila (2.5\% prevalence in A. muciniphila). In all 55 positive cases, these genes were found on contigs larger than the plasmid ( $\sim 8 \mathrm{~kb})$, suggesting that they may be integrated into the bacterial genome (as also reported in [19]). Of note, the A. muciniphila type strain $\mathrm{Muc}^{\mathrm{T}}$ carries no antibiotic resistance genes and its use does not raise any antibiotic resistance concern as also indirectly confirmed by dose scaling pilot studies in humans and toxicological studies in rabbit and other model organisms $[8,55]$; however, ongoing and future human trials with strains different from the type strain should carefully consider their antibiotic resistance potential. In conclusion, although the rare occurrence of antibiotic resistance genes from plasmid RSF1010 in some A. muciniphila genomes has evident safety implications for their use in therapeutic applications, our findings indicate that Akkermansia candidate species mostly lack genetic means to defend themselves against currently used antibiotics.

\section{Vitamin B12 synthesis capabilities were independently lost by two Akkermansia candidate species}

Due to its essential nature and limited availability in the human gut, vitamin B12 (cobalamin) is regarded as a key element in host-microbe interactions [56]. In a recent study, 75 Akkermansia strains were reported to differ in their potential to produce this important cofactor [21]. We set out to characterize the vitamin B12 synthesis capabilities of the Akkermansia candidate species as well as A. glycaniphila. By identifying corrin ring biosynthesis genes as a proxy for vitamin B12 synthesis capability [56], we confirm that the large majority of MAGs from candidate species SGB9227 and SGB9228 encode most proteins involved in producing vitamin B12 (75\% of SGB9227 MAGs encode all proteins except CbiA; 92\% of SGB9928 MAGs encode all proteins except $\mathrm{CbiF}$ ), while those genes were never found in A. muciniphila, SGB9223, nor SGB9224 (Fig. 2E). Interestingly, the more phylogenetically distant $A$. glycaniphila Pyt $^{\mathrm{T}}$ 
[22] also contains 6 out of 8 corrin ring biosynthesis genes (Fig. 2E). The differential vitamin B12 synthesis capabilities of Akkermansia spp. were successfully validated by in vitro assays: propionate production (a proxy for vitamin B12 production, as the pathway includes the B12-dependent methyl-malonyl CoA synthase reaction [57]) was detected when growing A. glycaniphila $\mathrm{Pyt}^{\mathrm{T}}$ but not $A$. muciniphila $\mathrm{Muc}^{\mathrm{T}}$ in the absence of vitamin B12 (Fig. 2F, Additional file 2: Figure S6). The cbiA gene that we did not detect in the majority of SGB9227 MAGs is not found in A. glycaniphila Pyt ${ }^{\mathrm{T}}$ either, suggesting that this gene may not be necessary for B12 production in Akkermansia spp. Furthermore, we detected a strong correlation between pairwise genetic distances of corrin ring biosynthesis genes and core genes between strains of SGB9227, SGB9228, and the singular A. glycaniphila genome (Spearman rho $=0.6, P$-value $<0.001$; Fig. $2 \mathrm{G}$ ), suggesting that the B12 biosynthesis genes are ancestral to all Akkermansia candidate species and were lost by A. muciniphila, SGB9223, and SGB9224 candidate species in the human gut. The most likely evolutionary scenario would consist of two independent loss events: one after the most recent common ancestor of SGB9223/SGB9224 separated from the ancestor of the remaining candidate species, and another after the ancestor A. muciniphila separated from the ancestor of SGB9228 (Additional file 2: Figure S7). Taken together, these results reveal two independent B12 synthesis loss events in Akkermansia candidate species and indicate that new Akkermansia strains should be studied for their potential to increase colonic vitamin B12 biosynthesis.

\section{Akkermansia candidate species encode a novel variant of type I-C CRISPR-Cas loci}

CRISPR-Cas systems are widely used by prokaryotes to fend off foreign DNA [58] and can be exploited to alter the microbiome makeup [59]. However, they have only been studied in detail for a limited number of bacteria, and strain-level variations have been documented [59]. We thus screened our catalog of Akkermansia genomes and MAGs for the presence of CRISPR-Cas loci. A great majority of genomes (68\%, Fig. 3A) harbored at least one CRISPR-Cas locus, and while type I-C loci [61] were detected in all Akkermansia candidate species, A. muciniphila is the only species in the genus sometimes carrying a type II-C locus (33\%, Fig. 3A). In 9\% of the cases, A. muciniphila strains carried both the type II-C locus and the type I-C locus (Fig. 3A).

The structure of type I-C loci in Akkermansia candidate species differs notably from the canonical organization [61]: Cas3, Cas5, Cas8c, and Cas7 genes are encoded in the opposite direction of Cas4, Cas1, and Cas2, thus representing a novel variant of type IC CRISPR-Cas loci. The majority of loci (62.4\%) contain two CRISPR arrays, one upstream and one downstream of the Cas gene cassette. In contrast, the type II-C loci of A. muciniphila have the canonical structure [62] in $95 \%$ of the strains in which the locus was detected (Fig. 3B). The presence of a type II-C locus in A. muciniphila has no clear phylogenetic structure (Fig. 3C), highlighting a peculiar evolutionary history. Akkermansia candidate species also differ in the total number of spacer sequences encoded in each genome as well as the fraction of loci that contain two (instead of one) CRISPR arrays (Fig. 3B, D). SGB9223 and SGB9228 on average contain more spacer sequences (median 43 s.d. 15.4 and median 36 s.d. 29.5) compared to SGB9224, which has the lowest number of spacers (median 3, s.d. 16.4). Similarly, 91\% of all genomes from SGB9223 contain two CRISPR arrays (one upstream, one downstream of the Cas 


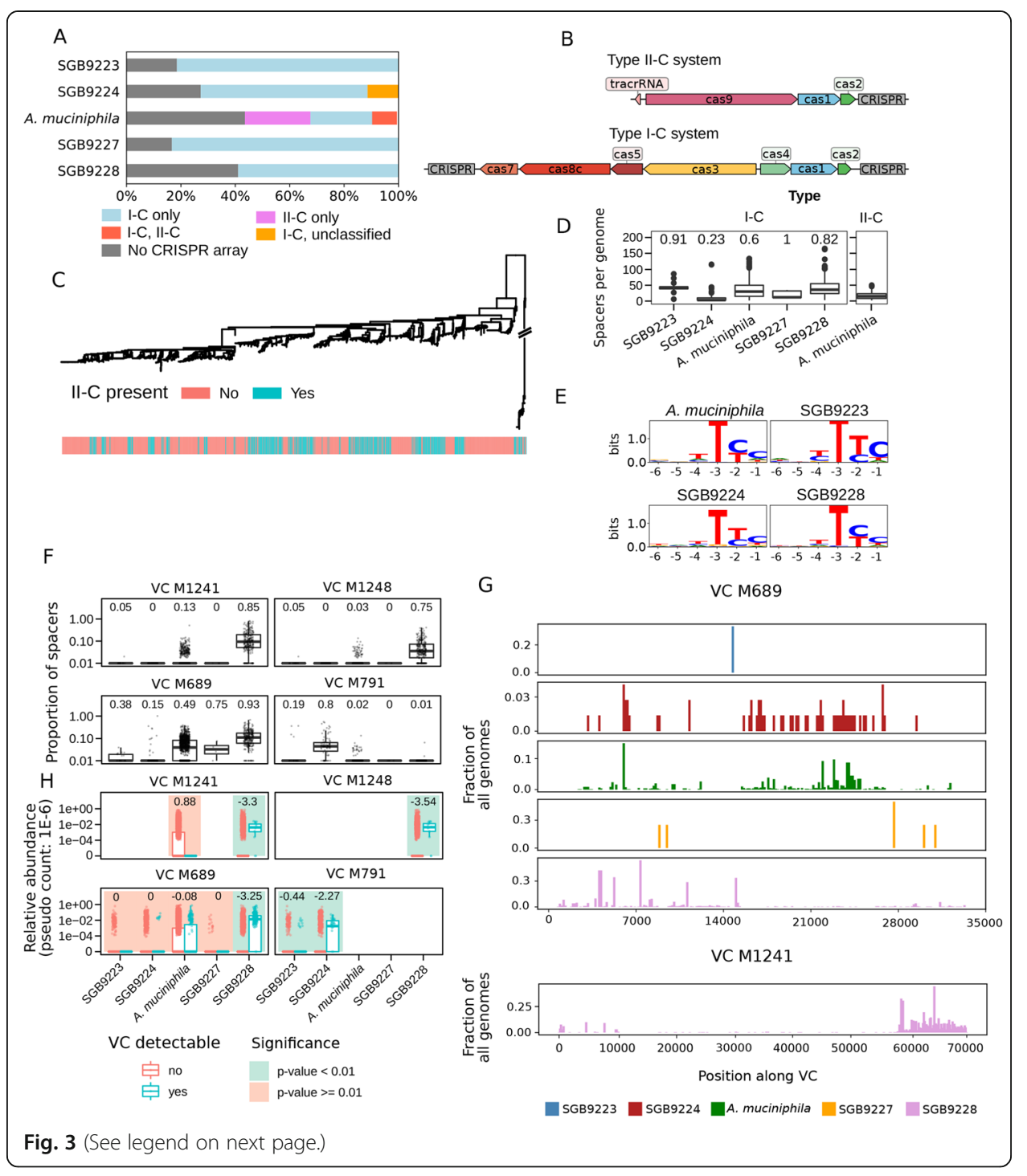


(See figure on previous page.)

Fig. 3 The CRISPR-Cas system of Akkermansia candidate species and their viral targets. A CRISPR locus type composition of Akkermansia candidate species. All candidate species possess CRISPR locus type I-C, with the exception of A. muciniphila in which type II-C is present in more than $30 \%$ of the genomes. B Representative locus organization of CRISPR loci over Akkermansia candidate species. Some type I-C loci contain only one CRISPR array. Gene and CRISPR array lengths are scaled to correspond to the median length over all loci. C Phylogenetic tree of A. muciniphila subspecies colored by type II-C presence. D The total number of spacer sequences for the genomes in each Akkermansia candidate species. Type II-C loci were only found in A. muciniphila. Numbers above the boxplots correspond to the fraction of type I-C loci with two CRISPR arrays. E Logo plots of predicted PAM sequences in putative (phage) Viral Clusters (VCs, see the "Methods" section) upstream of sequences with perfect matches against CRISPR spacer sequences from type I-C loci. F Proportion of CRISPR spacers within candidate species genomes with a near-perfect match (at most 2 mismatched nucleotides) for four VCs. The number above the box plots corresponds to the fraction of genomes with at least one spacer hit against a given VC (see the "Methods" section). G Mapping of spacers from Akkermansia genomes against two representative VCs, visualized with a sliding window of $150 \mathrm{nt}$. See Additional file 2: Figure S8 for the remaining VCs. $\mathbf{H}$ Distribution of the relative abundances of the Akkermansia candidate species based on the presence or absence of each cognate VC in the metagenome (Additional file 1: Table S2, see the "Methods" section). P-values for differential abundance were determined via two-sided Wilcoxon rank-sum tests. $P$-values of $<0.01$ were considered significant. The numbers above the box plots correspond to the generalized fold change, with negative numbers indicating a higher bacterial abundance when a VC is detected [60]

gene cassette), whereas only $23 \%$ of genomes in SGB9224 do so (Fig. 3D). Akkermansia candidate species thus generally contain CRISPR-Cas systems, with distinct loci structure and spacer composition, indicating considerable divergence in their exposure to foreign DNA over their evolutionary trajectory.

\section{Newly discovered putative phages are recognized by Akkermansia CRISPR-Cas systems and tend to co-occur with cognate candidate species}

We next identified de novo assembled, putative intestinal bacteriophages in shotgun gut viromes defining Viral Clusters (VCs, see the "Methods" section) and screened them for the presence of Akkermansia CRISPR-Cas spacers. We found no spacer hits against any of the known intestinal phages currently in RefSeq [63], but we instead detected a total of eight VCs with spacer hits (Additional file 2: Figure S8), four of which consistently attracted spacer sequences from at least one Akkermansia candidate species (Fig. 3F, see the "Methods" section) which we considered for further analysis. While some VCs exhibited hits from spacers from only one of the candidate species (SGB9228 for M1241 or M1248), other VCs (M689) were found to attract spacer sequences from all candidate species. Regardless of VCs, SGB9228 genomes on average have the highest total fraction of spacer sequences hit (Additional file 2: Figure S9).

The mapping of Akkermansia spacer sequences against VCs revealed that spacer sequences tend to cluster locally in the phage genome and that different locations on the viral genome attract spacers in a species-dependent fashion (Fig. 3G, Additional file 2: Figure S8). Furthermore, identification of the sequences directly upstream of all spacer sequence hits allowed reconstruction of the canonical type I-C protospacer adjacent motif (PAM) "TTC" (Fig. 3E) found in Bacillus halodurans [64]. The presence of multiple, distinct hits for some species-VC combination (Fig. 3G, Additional file 2: Figure S8) suggests that these matches are not spurious and that many combinations of Akkermansia candidate species and viral clusters reflect multiple bacterium-phage interactions in the intestinal environment. To further investigate potential ecological 
interactions between Akkermansia candidate species and phages, we assessed the cooccurrence between candidate species and the matching VCs across 13,237 metagenomes (see the "Methods" section). For 5 out of 10 putatively interacting pairs of VCs and candidate species (defined as those pairs where more than $10 \%$ of genomes of the candidate species have at least one VC-matching spacer), we found that a candidate species is significantly more abundant $(P$-value $<0.01)$ whenever the cognate $\mathrm{VC}$ is detectable (Fig. 3F, H, see the "Methods" section). Taken together, our analysis showed that CRISPR spacer sequences found in the genome of Akkermansia candidate species can be frequently mapped to four putative phages that co-occur with their cognate candidate species, suggesting that they are ecologically interacting in the human gut.

\section{A. muciniphila is stratified in four subspecies with different host preferences and functional profiles}

In all bacterial species, a large fraction of the phenotypic variability is encoded at the subspecies level $[5,14-16]$. We thus further focused on the intra-species genetic variation of $A$. muciniphila given its prevalence and relevance due to its association with lower host BMI (Fig. 2D). We found A. muciniphila to have four monophyletic subclades that we labeled Amuc1 to Amuc4 (Fig. 4A). We left strains unassigned that are not part of one of these monophyletic subclades (accounting for $29 \%$ of all $A$.

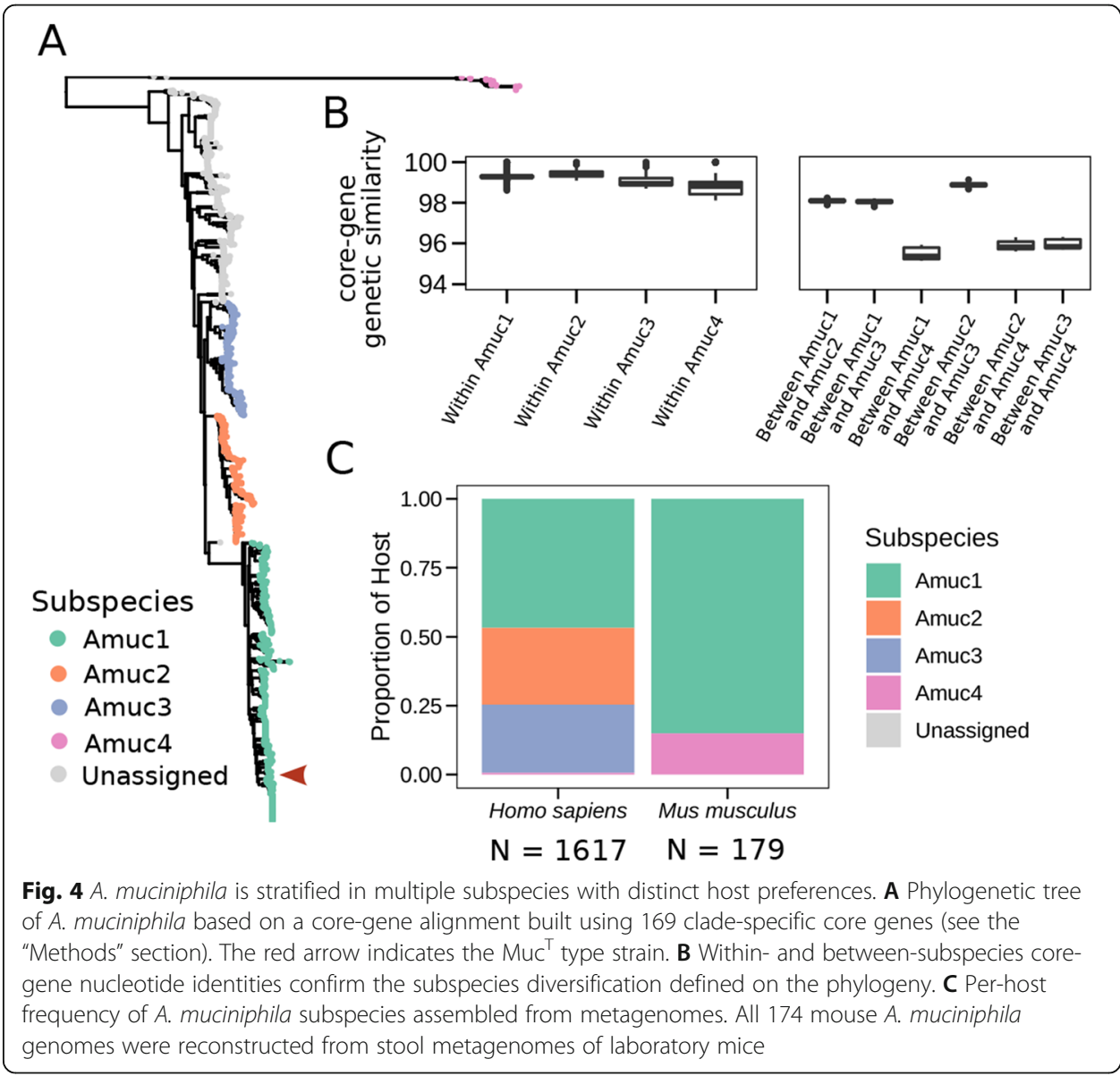


muciniphila strains). The subspecies were found to have similar within-subspecies genetic similarities-always exceeding 98\% identity-while between-subspecies genetic similarities range from $95.4 \%$ genetic similarity between Amuc1 and Amuc4 to 98.6\% between the more closely related Amuc2 and Amuc3 (Fig. 4B). This inter-subspecies genetic divergence was coupled also with a diversification of the functional profiles of the strains (Fig. 5A).

Amuc1 is the most prevalent subspecies in humans (47\%), followed by Amuc2 and Amuc3 (27\% and 24\% respectively, Fig. 4C). To investigate whether these global prevalences were driven by particular host factors, we studied the distribution of A. muciniphila subspecies across host metadata (Additional file 2: Figure S10). In addition to a significantly higher prevalence of Amuc4 in non-Westernized human populations compared to non-Amuc4 (Fisher test $P$-value $<0.001$ ), we found that subspecies were differentially distributed across hosts. In particular, Amuc2 and Amuc3 are specific to humans and never found in mice and non-human primates, whereas Amuc1 and Amuc4 can be found in both humans and mice (Fig. 4C) but in different proportions, suggesting differential fitness of A. muciniphila subspecies in mice compared to humans. Notably, all A. muciniphila genomes we obtained from mice came from laboratory-held mice. Due to the lack of subspecies-specific marker genes, we were unable to extend prevalence analysis to samples lacking successfully reconstructed Akkermansia MAGs, but our data nonetheless suggests Akkermansia in mice may be acquired from humans and that there is a strong preference of laboratory mice to acquire only the Amuc1 (to which

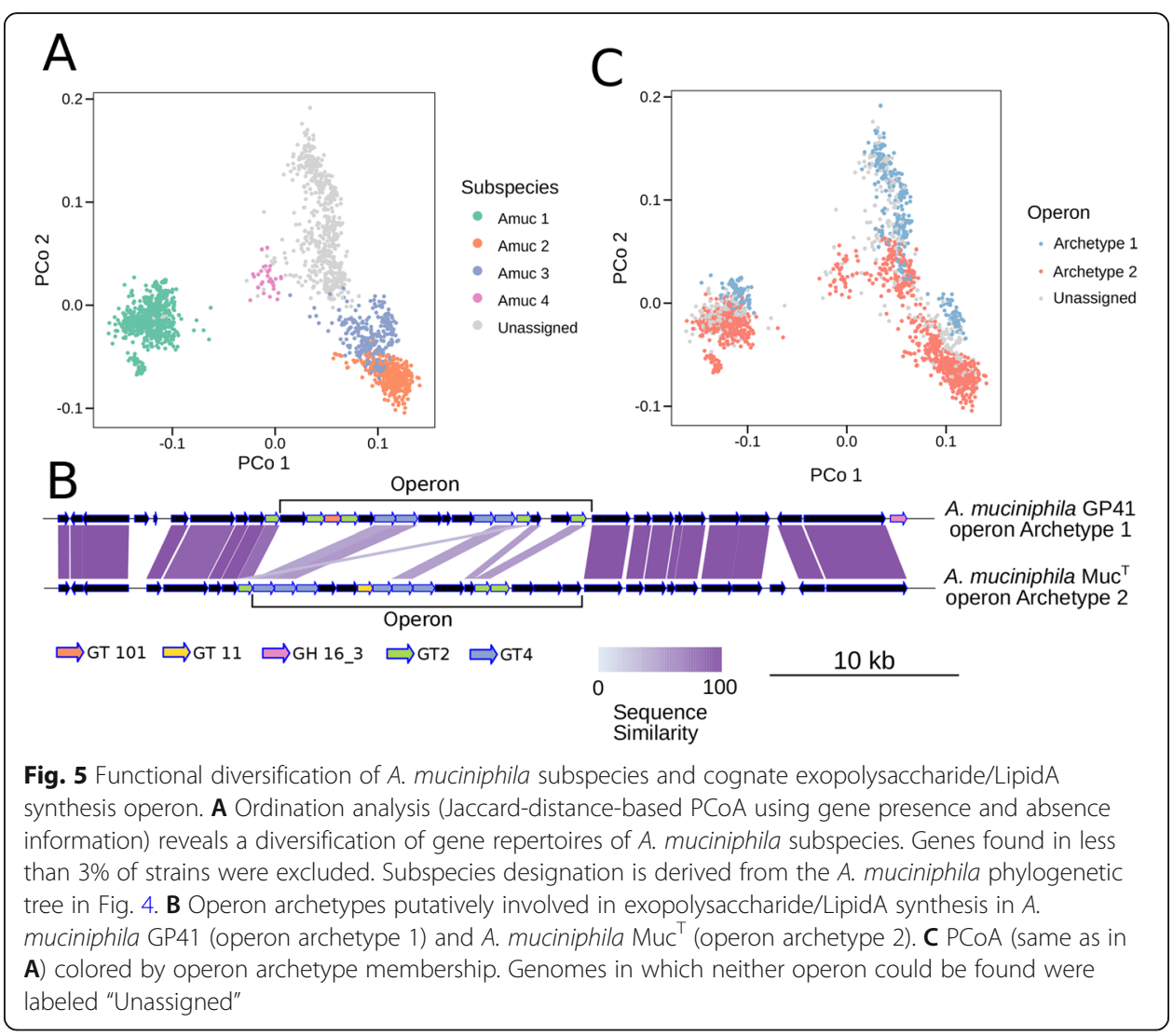


Muc ${ }^{\mathrm{T}}$ belongs) and Amuc4 A. muciniphila subspecies, which might have important implications for pre-clinical mice models.

Two functionally related but distinct glycosyltransferase-rich operons are found in the $A$. muciniphila subspecies

Surface glycoconjugates are known to form a species- and sometimes even strainspecific glycan barcode, conferring bacteria with unique interaction properties [65]. Two well-conserved archetypes of a glycosyltransferase-rich operon were detected in the same genomic location in different A. muciniphila strains (Fig. 5B, Additional file 2: Figure S11). Both operon archetypes predominantly contain genes annotated as glycosyltransferases (GTs) belonging to two different CAZyme families (GT2 and GT4), albeit in different proportions: while archetype 1 contains five GT2 and four GT4 copies and archetype 2 contains three GT2 and six GT4 copies. GTs belonging to these families are typically involved in lipo- and/or exopolysaccharide biosynthesis, which are key in microbiota-host interactions [65]. However, despite both operon archetypes being mostly composed of functionally related GTs, only a few pairs of proteins displayed detectable but very remote sequence similarity (Fig. 5B). The two operon archetypes were notably differentially distributed among $A$. muciniphila subspecies: subspecies Amuc2 and Amuc4 always possessed archetype 2 (whenever detectable), whereas strains belonging to Amuc1 and Amuc3 had either archetype. A. muciniphila thus encodes one of two possibly very distantly related operons that are putatively involved in lipo/exopolysaccharide (LPS/EPS) biosynthesis functions, hinting at a possible divergence of their surface glycoconjugates as well as host-specific selective advantages.

\section{Discussion}

The possibility of extracting whole (draft) microbial genomes of sufficient quality directly from metagenomic sequences $[3,9,24-26,66]$ together with the quickly increasing availability of metagenomes from diverse populations [67] is revolutionizing the way human-associated microbes can be studied and characterized [30-33]. Exploiting a combined set of over 18,600 metagenomic samples from multiple hosts, we studied the population genomics and genetic characteristics of bacterial strains belonging to the Akkermansia genus. While A. muciniphila is recognized as a keystone species of the human microbiome, current biomedical and translational research is still driven by the type strain $\mathrm{Muc}^{\mathrm{T}}$ [4], thus neglecting the genomic and phenotypic variability of conspecific strains as well as of closely related species. Previous comparative genomic efforts were able to survey only a fraction of the diversity in the Akkermansia genus we describe here due to the limited availability of isolate genomes [19, 20, 39]. At the same time, we extended similar ongoing work using MAGs for this genus [68] with a larger genome set and more diverse metagenomic sample set including non-human hosts, allowing us to explore aspects such as the association of Akkermansia abundances with phenotypes (particularly with respect to BMI), the in-depth analysis of some of its genetic features such as the machinery for vitamin B12 synthesis and a novel LPS/EPS operon, and the discovery of bacteriophages likely interacting with Akkermansia in the human gut. 
Our analysis of 2420 Akkermansia genomes delineates four candidate species in addition to the well-defined $A$. muciniphila species. The five candidate species are prevalent in the human gut microbiome and are found in other mammals such as mice and non-human primates almost exclusively when living in man-made environments, suggesting that all Akkermansia candidate species are specifically adapted to the human gut. All candidate species have very high pairwise sequence similarity of the full-length 16 S rRNA gene (>98\%) and substantially lower whole-genome similarity $(<90 \%$ for all pairs except SGB9223 and SGB9224). These unusual genomic characteristics are likely the reason why the diversity of the Akkermansia genus has been overlooked by extensive 16S rRNA gene amplicon sequencing surveys in the past. Most bacterial species at $<95 \%$ genomic similarity have $>3 \%$ divergence of the $16 \mathrm{~S}$ rRNA gene [3], and the substantially different pattern observed in Akkermansia might suggest rapid genomic diversification of these clades in humans.

A potential instance of adaptive evolution in Akkermansia emerging from our analysis could be the loss of vitamin B12 synthesis capabilities that likely occurred independently in the ancestors of two candidate species. Vitamin B12 promotes symbiotic metabolic relationships between gut microbes [56]. Notably, a bidirectional syntrophy has been described between A. muciniphila $\mathrm{Muc}^{\mathrm{T}}$ and Anaerobutyricum soehngenii (formerly known as Eubacterium hallii [69]), with $\mathrm{Muc}^{\mathrm{T}}$ converting mucin into oligosaccharides and acetate which are used by the butyrate-producer Anaerobutyricum soehngenii, in turn providing (pseudo)vitamin B12 to enable propionate production by $\operatorname{Muc}^{\mathrm{T}}$ [70]. Hence, loss of vitamin B12 synthesis genes might have been metabolically favorable for Akkermansia candidate species SGB9223/9224 and A. muciniphila given the potential to syntrophically interact in this way with other species. Our results warrant future investigations also at the level of subspecies clades: for example, the presence of one of the two putative LPS/EPS operons we described in A. muciniphila may be driven by host-microbe interactions and host-specific factors such as diet or lifestyle.

Experimental efforts to investigate Akkermansia-host interactions that are currently fueled by findings of their potential role in biomedical settings (ranging from obesity [5-8] to cancer treatments [71, 72]) should consider some aspects of the genus-wide genomic diversity we are reporting here. For example, only A. muciniphila was confirmed in our analysis to be associated with decreased BMI and it is possible that the $A$. muciniphila subspecies might also display different strengths of association. Moreover, the limitations of animal-based experimental approaches should be particularly considered for Akkermansia: our finding that no Akkermansia candidate species is consistently detected in wild mice and primates may indeed suggest that these animals are not natural hosts for Akkermansia, and raises the question whether host-Akkermansia interactions can be meaningfully recapitulated in mice. Similarly, we obtained MAGs from only two out of four A. muciniphila subspecies from mice, suggesting that not all subspecies may be well adapted to the mouse gut, which has important implications for in vivo experiments. Further delineation of subspecies through bacterial isolation or single-cell sequencing will be required to answer this question conclusively.

The ecology of Akkermansia may however be driven not only by aspects of host fitness, as interaction with bacteriophages also potentially contribute to shaping the population structure and diversity of this microbe. While no known phages have been so far 
linked with Akkermansia as a host, we identified at least four putative phages from gut viromes and gut metagenomes that display genomic regions recognized by spacer sequences in CRISPR-Cas loci in a species-specific manner. These putative phages also tend to co-occur with their cognate candidate species. Understanding the mechanisms of interaction between these phages and their targets could be an important experimental step in order to develop intervention strategies to modulate the presence and abundance of Akkermansia candidate species in the gut.

Our work provides a large-scale strain-level analysis of Akkermansia that can be the basis for future further investigations of this genus. We also further highlighted the potential of metagenomics-based investigations of bacteria of the human microbiome, which is particularly important given that most bacterial species have very little genomic information available from cultivation efforts. In our work, we also introduced new analysis types for MAG-based investigations complementing those already performed on other bacteria such as Eubacterium rectale [31], Prevotella copri [30], Ruminococcus gnavus [32], and Faecalibacterium prausnitzii [33]. Further extending and applying this approach to the hundreds of species in the human microbiomes will be crucial to better understand the bacterial constituents of human-associated microbial ecosystems.

\section{Material and methods}

\section{Collection and taxonomic annotation of Akkermansia sp. genomes}

The Akkermansia genomic sequences used in this work were retrieved from four sources: (i) newly sequenced Akkermansia genomes from cultivated strains [43], (ii) publicly available isolated genomes from NCBI (downloaded as of March 2020) that were labeled as Akkermansia muciniphila or Akkermansia sp., (iii) metagenomeassembled genomes (MAGs) coming from a collection of metagenomes from human microbiome by Pasolli et al. [3], and (iv) 166,518 additional MAGs reconstructed from 9172 metagenomes (Additional file 1: Table S4) obtained with a validated assemblybased pipeline similarly to Pasolli et al. [3].

For the 166,518 additional MAGs reconstructed specifically for this work, the metagenomes were assembled using metaSPAdes [73] if paired-end metagenomes were available, and MEGAHIT [74] otherwise. In both cases, default parameters were used. Contigs longer than 1500 nucleotides were binned into MAGs using MetaBAT2 [75]. We assigned MAGs to previously defined species-level genome bins (SGB) (Pasolli et al. [3]) based on whole-genome nucleotide similarity estimation using Mash [76] and only MAGs falling in the SGBs belonging to the Akkermansiaceae family were further considered. We then quality controlled the MAGs and genomes using checkM (version 1.1.3) [42] and kept genomes estimated to be high-quality according to genomic completeness $>90 \%$ and genomic contamination $<5 \%$.

The above procedure resulted in a total of 2420 Akkermansia genomes being considered in this work (http://segatalab.cibio.unitn.it/data/Akkermansia_Karcher_et_al.html): 188 isolate genomes from NCBI (119 labeled as Akkermansia muciniphila and 69 labeled as Akkermansia sp.), 2226 MAGs, and 6 novel genomes coming from strains isolated from the human gut. The 2420 genomes were assigned to a total of five candidate species which includes the already recognized Akkermansia muciniphila species and 
four additional SGBs: SGB9223, SGB9224, SGB9227, and SGB9228 as summarized in Table 1.

\section{Identification and comparison of the 16S rRNA genes from genomes and MAGs}

$16 \mathrm{~S}$ rRNA genes were identified using Barrnap (version 0.9) with default parameters. We only considered extracted $16 \mathrm{~S}$ rRNA gene sequences longer than 1000 nucleotides. We retained a total of 445 16S rRNA sequences (255 from isolate genomes and 190 from MAGs). Mapping all these sequences against the NCBI's 16S rRNA gene set identified 11 outlying $16 \mathrm{~S}$ rRNA genes that had $>98 \%$ whole-gene identity to a $16 \mathrm{~S}$ rRNA gene of a family other than Akkermansiaceae, which we removed. We then aligned the sequences using mafft (version v7.471, [77]) with parameters: --quiet --anysymbol --localpair --maxiterate 1000) and computed pairwise edit distances between all sequences.

\section{Genome annotation and gene clustering}

We detected and annotated ORFs on all genomes using Prokka (version 1.14) [78]. Coding sequences (CDS) were then assigned to a UniRef90 cluster [79] by performing a Diamond search (version 0.9.24) [80] of the CDS against the UniRef90 database (version 201906) and assigning a Uniref90-ID if the mean sequence identity to the centroid sequence is over $90 \%$ and if it covers more than $80 \%$ of the centroid sequence. Protein sequences that could not be assigned to any UniRef90 cluster were de novo clustered using MMseqs2 [81] following the Uniclust90 criteria [82].

\section{Whole-genome phylogenetic analysis}

The phylogenetic analyses were performed with PhyloPhlAn3 [46], using either 400 universal marker genes when applied on the 2420 Akkermansia genomes or core genes when applied to each separate candidate species. Core genes of an Akkermansia candidate species were those ORFs whose assigned UniRef90 annotation (or de novo clustered gene family) was present in at least $80 \%$ of the genomes of the candidate species. The number of core genes varied across candidate species, with 1131 for SGB9223, 799 for SGB9224, 996 for SGB9228, and 169 for A. muciniphila. The phylogenies were obtained using PhyloPhlAn 3.0 using the following flags, in both cases, universal markers and specific core genes: "--force_nucleotides --trim greedy--fast--diversity low". The following tools with their specific parameter are used inside the PhyloPhlAn3 framework, diamond was used over blast to generate the database when the database sequences were in proteins:

Table 1 Summary of the number of genomes per candidate species

\begin{tabular}{lllllll}
\hline & SGB9223 & SGB9224 & A. muciniphila & SGB9227 & SGB9228 & Total \\
\hline MAGs & 29 & 93 & 1802 & 4 & 298 & 2226 \\
Isolate genomes (NCBI) & 66 & 3 & 108 & 2 & 9 & 188 \\
Isolate genomes (generated) & 0 & 0 & 6 & 0 & 0 & 6 \\
Total & 95 & 96 & 1916 & 6 & 307 & 2420 \\
\hline
\end{tabular}


- diamond (version v2.0.2.140, [80]) with parameters: makedb (to generate the database), "blastx --quiet --threads 1 --outfmt 6 --more-sensitive --id 50 --max-hsps 35 - $k 0$ " (to map the dna) and "blastp --quiet --threads 1 --outfmt 6 --more-sensitive --id 50 --max-hsps $35-k 0$ "

- blast (version 2.10.1+, [83, 84]) with parameters: "makeblastdb -parse_seqids -dbtype nucl and blastn -outmft 6 -max_target_seqs 1000000"

- Mafft (version v7.471, [77]) with parameters : “--quiet --anysymbol --localpair --maxiterate 1000"

- trimal (v1.4.rev15 build[2013-12-17], [85]) with parameters: ":-gappyout"

- RAxML (version 8.2.12, [86]) with parameters: “- $p$ 1989 -m GTRCAT -x 1989 -\# $100-f a^{\prime \prime}$

\section{Relative abundance estimation of candidate species}

In order to estimate the presence and relative abundance of the Akkermansia candidate species, we extended the database of unique marker genes of MetaPhlAn $3.0[49,87]$ with those of the newly defined Akkermansia candidate species. Unique marker genes were defined starting from the core genes of each of the 5 Akkermansia candidate species identified on the clustered gene families described above. Core genes of each Akkermansia candidate species were divided into 150 nucleotide fragments and then aligned against the genomes of all SGBs including both the other Akkermansia candidate species as well as the whole set of bacterial and archaeal SGBs defined in Pasolli et al. [3] using bowtie2 (version 2.3.5.1; --sensitive option) [88]. A core gene was considered present in a genome if at least one of the gene's fragments was mapping against it. Core genes never found in more than $1 \%$ of the sequences included in any other SGBs were selected as marker genes, obtaining 39, 22, 115, 100, and 135 speciesspecific unique markers for SGB9223, SGB9224, SGB9227, SGB9228, and A. muciniphila, respectively. MetaPhlan 3 was then used with default parameters. The prevalence of candidate species was defined as the percentage of samples in which the candidate species was detected. Similarly, the prevalence of the Akkermansia genus was defined as the percentage of samples in which at least one of the candidate species could be detected.

\section{Covariation among candidate species}

Covariation among relative abundances of Akkermansia candidate species was assessed in 4171 human metagenome samples in which at least one of the candidate species was detected (out of the 11,014 metagenomes from humans, Additional file 1: Table S2) by performing pairwise Spearman's correlations (cor.test in the stats R package [89]). We corrected for multiple testing using the Benjamini-Hochberg procedure at 10\% FDR.

\section{Association between candidate species and metadata parameters}

The association between relative abundances of Akkermansia candidate species and host BMI, age, and gender was analyzed in 3311 human metagenomic samples from 22 datasets in which this information was available (Additional file 1: Table S3). For continuous variables (age and BMI), Spearman's correlations were computed using the pcor.test function from the ppcor $\mathrm{R}$ package [90] controlling for the remaining 
covariates. Resulting correlations were used as input in the metacor function from the meta $\mathrm{R}$ package [91] using Fisher's Z transformation of correlations and the PauleMandel estimator of between-study variance in the random effects model. For categorical variables (sex), an ordinary least squares (OLS) model was first used to adjust for age and BMI. Statistical significance (Wald test) and effect sizes (standardized mean difference) of the associations were extracted from the sex beta coefficients. Resulting effect sizes were inverse-variance averaged using the Paule-Mandel estimator of between-study variance as implemented in the statsmodels python library [92] and custom code. We corrected for multiple testing using the Benjamini-Hochberg procedure at $10 \%$ FDR.

\section{Identification of corrin ring biosynthesis genes}

Anaerobic corrin ring biosynthesis gene names were obtained from [93]. Corresponding KEGG Orthologs (KOs) were then identified in the clustered gene sequences (see above) using KOFAM [94]. Only those hits that passed the optimized bit-score cutoffs from KOFAM were considered. We found a total of 316 genomes with at least one significant hit.

\section{Determination of vitamin B12 utilization and production by $A$. muciniphila and $A$. glycaniphila}

The type strains A. muciniphila Muc ${ }^{\mathrm{T}}$ (ATCC BAA-835) and A. glycaniphila Pyt ${ }^{\mathrm{T}}$ (DSM 100705) were grown in minimal bicarbonate buffered medium supplemented

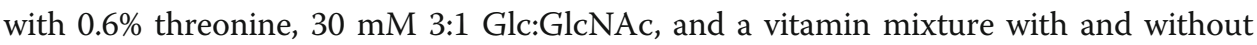
added vitamin B12 [95]. Cultures were inoculated with a preculture produced on mucin-supplemented medium. At several time points $(0,3,8.5,21,28,33,48 \mathrm{~h})$, a 1 $\mathrm{mL}$ sample was collected to measure cell density (OD 600nm) and determine propionate concentration as a proxy for vitamin B12 production. Substrate utilization and metabolite production were quantified on a Thermo Scientific HPLC system equipped with an Agilent Metacarb $67 \mathrm{H} 300 \times 6.5 \mathrm{~mm}$ column. The column was kept at $45^{\circ} \mathrm{C}$, running $0.005 \mathrm{M} \mathrm{H}_{2} \mathrm{SO}_{4}$ eluent at a flow rate of $1 \mathrm{~mL} / \mathrm{min}$. Detection was performed using a refractive index detector. All measurements were performed in duplicate.

\section{LPS operon identification}

The pangenome of $A$. muciniphila was reconstructed using the UniRef90 assignments and complemented with the de novo clustered gene families (see above). Pan-genes were then also annotated with CAZy using a local dbCAN distribution [96] (database version V9 with suggested E-value and HMM coverage cutoffs of $1 \mathrm{E}-18$ and 0.35 , respectively). We specifically focused on the differential copy number and distribution of the glycosyltransferase enzymes class 2 and 4 (GT2/GT4) in the A. muciniphila genomes. We observed two groups within this set of genes that were co-present and mutually exclusive in genomes, suggesting a large structural variation and operon-type distribution of genes. We then determined the two putative archetypes by manual inspection of gene distribution and order on isolate genomes. Finally, the detected dichotomy was confirmed by performing BLAST on operon genes (including bordering 
genes from the isolate genomes) against all genomes and observing their presence/absence (Additional file 2: Figure S11).

\section{CAZy annotation and gene clustering}

dbCAN2 ([97], database version 07312020) was used to annotate centroid sequences of gene clusters (see above) with Carbohydrate-Active enZYmes (CAZY) information [98]. dbCAN2 was used with default parameters, and hits with an E-value $>10 \mathrm{E}-15$ and those that covered less than 35\% of a given dbCAN2-HMM were removed.

\section{Retrieval of CRISPR spacers in viruses from metagenomes and viromes}

Metagenomes enriched for virus-like particles (i.e., viromes) were retrieved through SRA [99] from 708 samples from five studies [100-104]. Samples were uniformly preprocessed with TrimGalore version 0.4.4 [105] to remove low quality and short reads (Phred quality $<20$, read length $<75$; parameters: --stringency 5 --length 75 --quality 20 --max_n 2 --trim-n). Reads aligning to the human genome (hg19) were identified and subsequently removed via mapping with Bowtie2 version 2.4.1 [88] in global mode. Raw reads were assembled with metaSPAdes [73] version 3.10.1 (k-mer sizes: -k 21,33, $55,77,99,127)$. The efficacy of viral enrichment of each virome was evaluated with ViromeQC [106], and 126 out of 708 samples had an enrichment higher than 50-fold. Contigs (a) longer than 1500bp; (b) originating from highly enriched viromes (i.e., enrichment $\geq 50 x$ ); (c) found binned in the same Species-level Genome Bin [3] in less than 30 metagenomes; and (d) found in the unbinned fraction of more than 20 metagenomes [3] were retained as putative viral contigs. After this, contigs originating from non-highly enriched viromes with a high sequence similarity were added to the collection (BLAST identity $\geq 80 \%$, length $\geq 1000$ nucleotides, by using BLAST, version 2.6.0 [107]). Sequences homologous to the virome-derived contigs were searched in unbinned contigs of Pasolli et al. with mash version 2.0 [76], and contigs with a distance lower than $10 \%$ ( $p$-value $\leq 0.05)$ to any viral contig were added to the collection. Finally, we added 699 full genomes of taxonomically annotated gut bacteriophages from RefSeq, release 99 [63] that were also found in at least 20 metagenomes of the unbinned fraction of Pasolli et al. [3].

Putative viral contigs were then clustered at 70\% identity with VSearch version 2.14 .2 [108] (parameters --cluster_fast --id 0.7 --strand both) and further grouped if they shared more than one third of their sequence at $90 \%$ sequence identity or more to produce 1345 "Viral Clusters" (VCs) that were further analyzed.

CRISPR arrays and Cas genes were predicted using CRISPRCasTyper version 1.2.1 (default parameters) [62]. In order to understand potential interaction of candidate species and VCs, we aligned spacer sequences against VCs with BLAST version 2.2.31 (parameters -task blastn-short -gapopen 1 -gapextend 2 -penalty -1 -reward 1 -evalue 1 -word_size 10). Near-perfect matches were defined as matches with an edit distance $\leq$ 2. CRISPR-Cas loci structures were plotted using DNA Features Viewer version 3.0.3 [109]. Sequence logos were generated using Logomaker version 0.8 [110]. We used spacers from orphan as well as non-orphan CRISPR arrays for all spacer-based analyses (Fig. 3D, F-H). For subsequent analysis, we considered only those four VCs where at 
least $5 \%$ of the genomes of a given candidate species had at least one spacer sequence with a hit.

In order to detect the presence of a $\mathrm{VC}$ in a metagenome, we mapped a total of 13 , 381 gut metagenomes against VC contigs with Bowtie2 [88] version 2.4.1 in global mode. Breadth and depth of coverage were evaluated for each $\mathrm{VC}$ with bedtools version 2.29.1 [111] (genomecov command, default parameters). Only alignments with a Bowtie2 alignment score (AS:i tag) greater than -50 were considered. A VC was considered detected if at least one sequence in the cluster had a breadth of coverage of at least $50 \%$. Differential abundance of VCs in subspecies was assessed with two-sided Wilcoxon rank-sum tests. $P$-values of $<0.01$ were considered significant.

\section{Supplementary Information}

The online version contains supplementary material available at https://doi.org/10.1186/s13059-021-02427-7.

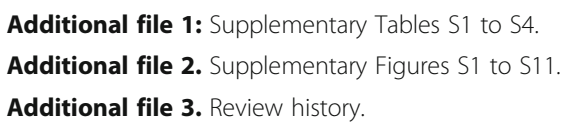

Peer review information

Kevin Pang was the primary editor of this article and managed its editorial process and peer review in collaboration with the rest of the editorial team.

\section{Review history}

The review history is available as Additional file 3

\section{Authors' contributions}

NS, WdV, MVC, and NK conceived and supervised the study. NK, EN, MP, ABM, PM, MZ, FC, and MVC performed the data acquisition. NK, EN, MP, ABM, MC, PM, MZ, FC, DG, SM, and MVC performed the data analysis. TPNB, HLPT, and WdV designed and performed the in vitro experiments. NK, EN, MC, AC, MA, MVC, WdV, and NS performed the data interpretation and wrote the manuscript. All authors read and approved the final manuscript.

\section{Funding}

This work was supported by the European Research Council (ERC-STG project MetaPG-716575) to NS, by MIUR 'Futuro in Ricerca' (grant no. RBFR13EWWI_001) to NS, by the European H2020 program (ONCOBIOME-825410 project and MASTER-818368 project) to NS, by the National Cancer Institute of the National Institutes of Health (1U01CA230551) to NS, and by the Premio Internazionale Lombardia e Ricerca 2019 to NS. This work was partly supported by the SIAM Gravitation Grant 024.002.002 and the 2008 Spinoza Award of the Netherlands Organization for Scientific Research to WMdV. We thank the Institute of Biotechnology, University of Helsinki (Finland), for providing both Illumina and PacBio sequences of new isolates.

\section{Availability of data and materials}

The 2420 Akkermansia genomes and MAGs considered in this work are available at http://segatalab.cibio.unitn.it/data/ Akkermansia_Karcher_et_al.html as well as in Zenodo under the following accession 0.5281/zenodo.5018705 [112].

\section{Declarations}

Ethics approval and consent to participate

Not applicable.

\section{Consent for publication}

Not applicable

\section{Competing interests}

WMdV is co-founder and holds stock in A-mansia Biotech Belgium. All the other authors declare that they have no competing interests.

\section{Author details}

${ }^{1}$ Department CIBIO, University of Trento, Trento, Italy. ${ }^{2}$ Novo Nordisk Foundation Center for Basic Metabolic Research, Faculty of Health and Medical Sciences, University of Copenhagen, Copenhagen, Denmark. ${ }^{3}$ Laboratory of Microbiology, Wageningen University, Wageningen, The Netherlands. ${ }^{4}$ Current address: Nestlé Institute of Health Sciences, Nestlé Research, Société des Produits Nestlé S.A., Lausanne, Switzerland. ${ }^{5}$ Human Microbiome Research Program, Faculty of Medicine, University of Helsinki, Helsinki, Finland. ${ }^{6} \mathrm{EEO}$, European Institute of Oncology IRCCS, Milan, Italy. 
Received: 18 December 2020 Accepted: 30 June 2021

Published online: 14 July 2021

\section{References}

1. Human Microbiome Project Consortium. Structure, function and diversity of the healthy human microbiome. Nature. 2012:486:207-14.

2. Qin J, Li R, Raes J, Arumugam M, Burgdorf KS, Manichanh C, et al. A human gut microbial gene catalogue established by metagenomic sequencing. Nature. 2010;464:59-65.

3. Pasolli E, Asnicar F, Manara S, Zolfo M, Karcher N, Armanini F, et al. Extensive unexplored human microbiome diversity revealed by over 150,000 genomes from metagenomes spanning age, geography, and lifestyle. Cell. 2019;176:649-62. e20.

4. Derrien M, Vaughan EE, Plugge CM, de Vos WM. Akkermansia muciniphila gen. nov., sp. nov., a human intestinal mucindegrading bacterium [Internet]. Int J Syst Evol Microbiol. 2004:1469-76 Available from: https://doi.org/10.1099/ijs.0.02 873-0.

5. Yang M, Bose S, Lim S, Seo J, Shin J, Lee D, et al. Beneficial effects of newly isolated Akkermansia muciniphila strains from the human gut on obesity and metabolic dysregulation [Internet]. Microorganisms. 2020:1413 Available from: https://doi.org/10.3390/microorganisms8091413.

6. de Vos WM. Microbe Profile: Akkermansia muciniphila: a conserved intestinal symbiont that acts as the gatekeeper of our mucosa. Microbiology. 2017;163:646-8.

7. Everard A, Belzer C, Geurts L, Ouwerkerk JP, Druart C, Bindels LB, et al. Cross-talk between Akkermansia muciniphila and intestinal epithelium controls diet-induced obesity. Proc Natl Acad Sci U S A. National Academy of Sciences. 2013;110: $9066-71$.

8. Depommier C, Everard A, Druart C, Plovier H, Van Hul M, Vieira-Silva S, et al. Supplementation with Akkermansia muciniphila in overweight and obese human volunteers: a proof-of-concept exploratory study. Nat Med. 2019;25:1096103.

9. Truong DT, Tett A, Pasolli E, Huttenhower C, Segata N. Microbial strain-level population structure and genetic diversity from metagenomes. Genome Res. 2017;27:626-38.

10. Scholz M, Ward DV, Pasolli E, Tolio T, Zolfo M, Asnicar F, et al. Strain-level microbial epidemiology and population genomics from shotgun metagenomics. Nat Methods. 2016;13:435-8.

11. Costea PI, Coelho LP, Sunagawa S, Munch R, Huerta-Cepas J, Forslund K, et al. Subspecies in the global human gut microbiome. Mol Syst Biol. 2017;13:960.

12. Schloissnig S, Arumugam M, Sunagawa S, Mitreva M, Tap J, Zhu A, et al. Genomic variation landscape of the human gut microbiome. Nature. 2013:493:45-50.

13. Van Rossum T, Ferretti P, Maistrenko OM, Bork P. Diversity within species: interpreting strains in microbiomes. Nat Rev Microbiol. 2020;18:491-506.

14. De Filippis F, Pasolli E, Tett A, Tarallo S, Naccarati A, De Angelis M, et al. Distinct genetic and functional traits of human intestinal Prevotella copri strains are associated with different habitual diets. Cell Host Microbe. 2019;25:444-53.e3.

15. Fehlner-Peach H, Magnabosco C, Raghavan V, Scher JU, Tett A, Cox LM, et al. Distinct polysaccharide utilization profiles of human intestinal Prevotella copri isolates. Cell Host Microbe. 2019:26:680-90.e5.

16. Maier L, Pruteanu M, Kuhn M, Zeller G, Telzerow A, Anderson EE, et al. Extensive impact of non-antibiotic drugs on human gut bacteria. Nature. 2018:555:623-8.

17. Yang C, Mogno I, Contijoch EJ, Borgerding JN, Aggarwala V, Li Z, et al. Fecal IgA levels are determined by strain-level differences in Bacteroides ovatus and are modifiable by gut microbiota manipulation. Cell Host Microbe. 2020;27:467-75. e6.

18. Geva-Zatorsky N, Sefik E, Kua L, Pasman L, Tan TG, Ortiz-Lopez A, et al. Mining the human gut microbiota for immunomodulatory organisms. Cell. 2017;168:928-43.e11.

19. Guo X, Li S, Zhang J, Wu F, Li X, Wu D, et al. Genome sequencing of 39 Akkermansia muciniphila isolates reveals its population structure, genomic and functional diverisity, and global distribution in mammalian gut microbiotas. BMC Genomics. 2017;18:800.

20. Xing J, Li X, Sun Y, Zhao J, Miao S, Xiong Q, et al. Comparative genomic and functional analysis of Akkermansia muciniphila and closely related species. Genes Genomics. 2019;41:1253-64.

21. Kirmiz N, Galindo K, Cross KL, Luna E, Rhoades N, Podar M, et al. Comparative genomics guides elucidation of vitamin B12 biosynthesis in novel human-associated Akkermansia strains. Appl Environ Microbiol. 2020;86 Available from: https:// doi.org/10.1128/AEM.02117-19.

22. Ouwerkerk JP, Aalvink S, Belzer C, de Vos WM. Akkermansia glycaniphila sp. nov., an anaerobic mucin-degrading bacterium isolated from reticulated python faeces. Int J Syst Evol Microbiol. 2016;66:4614-20.

23. Ouwerkerk JP, Koehorst JJ, Schaap PJ, Ritari J, Paulin L, Belzer C, et al. Complete genome sequence of Akkermansia glycaniphila strain PytT, a mucin-degrading specialist of the reticulated python gut. Genome Announc. 2017;5 Available from: https://doi.org/10.1128/genomeA.01098-16.

24. Nayfach S, Shi ZJ, Seshadri R, Pollard KS, Kyrpides NC. New insights from uncultivated genomes of the global human gut microbiome. Nature. 2019:568:505-10.

25. Almeida A, Mitchell AL, Boland M, Forster SC, Gloor GB, Tarkowska A, et al. A new genomic blueprint of the human gut microbiota. Nature. 2019;568:499-504

26. Almeida A, Nayfach S, Boland M, Strozzi F, Beracochea M, Shi ZJ, et al. A unified catalog of 204,938 reference genomes from the human gut microbiome. Nat Biotechnol. 2020; Available from: https://doi.org/10.1038/s41587-020-0603-3.

27. Costea PI, Munch R, Coelho LP, Paoli L, Sunagawa S, Bork P. metaSNV: a tool for metagenomic strain level analysis. PLoS One. 2017;12:e0182392.

28. Luo C, Knight R, Siljander H, Knip M, Xavier RJ, Gevers D. ConStrains identifies microbial strains in metagenomic datasets. Nat Biotechnol. 2015;33:1045-52.

29. Quince C, Delmont TO, Raguideau S, Alneberg J, Darling AE, Collins G, et al. DESMAN: a new tool for de novo extraction of strains from metagenomes. Genome Biol. 2017;18:181. 
30. Tett A, Huang KD, Asnicar F, Fehlner-Peach H, Pasolli E, Karcher N, et al. The Prevotella copri complex comprises four distinct clades underrepresented in westernized populations. Cell Host Microbe. 2019; Available from: https://doi.org/1 0.1016/j.chom.2019.08.018

31. Karcher N, Pasolli E, Asnicar F, Huang KD, Tett A, Manara S, et al. Analysis of 1321 Eubacterium rectale genomes from metagenomes uncovers complex phylogeographic population structure and subspecies functional adaptations [Internet]. Genome Biology. 2020; Available from: https://doi.org/10.1186/s13059-020-02042-y.

32. Hall AB, Yassour M, Sauk J, Garner A, Jiang X, Arthur T, et al. A novel Ruminococcus gnavus clade enriched in inflammatory bowel disease patients. Genome Med. 2017;9:103.

33. De Filippis F, Pasolli E, Ercolini D. Newly explored Faecalibacterium diversity is connected to age, lifestyle, geography, and disease. Curr Biol. 2020; Available from: https://doi.org/10.1016/j.cub.2020.09.063.

34. Barrangou R, Marraffini LA. CRISPR-Cas systems: prokaryotes upgrade to adaptive immunity. Mol Cell. 2014;54:234-44

35. Ogata Y, Sakamoto M, Ohkuma M, Hattori M, Suda W. Complete genome sequence of Akkermansia muciniphila JCM 30893, isolated from feces of a healthy Japanese male. Microbiol Resour Announc. 2020;9 Available from: https://doi. org/10.1128/MRA.01543-19.

36. Li H, Limenitakis JP, Fuhrer T, Geuking MB, Lawson MA, Wyss M, et al. The outer mucus layer hosts a distinct intestinal microbial niche. Nat Commun. 2015;6:8292.

37. Garzetti D, Brugiroux S, Bunk B, Pukall R, McCoy KD, Macpherson AJ, et al. High-quality whole-genome sequences of the oligo-mouse-microbiota bacterial community. Genome Announc. 2017;5 Available from: https://doi.org/10.1128/ genomeA.00758-17.

38. Medvecky M, Cejkova D, Polansky O, Karasova D, Kubasova T, Cizek A, et al. Whole genome sequencing and function prediction of 133 gut anaerobes isolated from chicken caecum in pure cultures. BMC Genomics. 2018;19:561.

39. Poyet M, Groussin M, Gibbons SM, Avila-Pacheco J, Jiang X, Kearney SM, et al. A library of human gut bacterial isolates paired with longitudinal multiomics data enables mechanistic microbiome research. Nat Med. 2019;25:1442-52.

40. Jiang $X$, Hall AB, Arthur TD, Plichta DR, Covington $C T$, Poyet $M$, et al. Invertible promoters mediate bacterial phase variation, antibiotic resistance, and host adaptation in the gut. Science. 2019;363:181-7.

41. Liu C, Zhou N, Du M-X, Sun Y-T, Wang K, Wang Y-J, et al. The Mouse Gut Microbial Biobank expands the coverage of cultured bacteria. Nat Commun. 2020;11:79.

42. Parks DH, Imelfort M, Skennerton CT, Hugenholtz P, Tyson GW. CheckM: assessing the quality of microbial genomes recovered from isolates, single cells, and metagenomes. Genome Res. 2015;25:1043-55.

43. Ouwerkerk JP. Akkermansia species: phylogeny, physiology and comparative genomics. 2016; Available from: https:// research.wur.nl/en/publications/akkermansia-species-phylogeny-physiology-and-comparative-genomics

44. Segata N, Börnigen D, Morgan XC, Huttenhower C. PhyloPhIAn is a new method for improved phylogenetic and taxonomic placement of microbes. Nat Commun. 2013:4:2304.

45. Zhu Q, Mai U, Pfeiffer W, Janssen S, Asnicar F, Sanders JG, et al. Phylogenomics of 10,575 genomes reveals evolutionary proximity between domains Bacteria and Archaea [Internet]. Nat Commun. 2019; Available from: https://doi.org/10.103 8/s41467-019-13443-4.

46. Asnicar F, Thomas AM, Beghini F, Mengoni C, Manara S, Manghi P, et al. Precise phylogenetic analysis of microbial isolates and genomes from metagenomes using PhyloPhlAn 3.0. Nat Commun. 2020;11:2500.

47. Jain C, Rodriguez-R LM, Phillippy AM, Konstantinidis KT, Aluru S. High throughput ANI analysis of 90K prokaryotic genomes reveals clear species boundaries. Nat Commun. 2018;9:5114.

48. Segata N, Waldron L, Ballarini A, Narasimhan V, Jousson O, Huttenhower C. Metagenomic microbial community profiling using unique clade-specific marker genes. Nat Methods. 2012;9:811-4.

49. Beghini F, Mclver LJ, Blanco-Miguez A, Dubois L, Asnicar F, Maharjan S, et al. Integrating taxonomic, functional, and strain-level profiling of diverse microbial communities with bioBakery 3 [Internet]. Cold Spring Harbor Lab. 2020:2020.11. 19.388223 [cited 2020 Nov 23]. Available from: https://www.biorxiv.org/content/10.1101/2020.11.19.388223v1.

50. Dao MC, Everard A, Aron-Wisnewsky J, Sokolovska N, Prifti E, Verger EO, et al. Akkermansia muciniphila and improved metabolic health during a dietary intervention in obesity: relationship with gut microbiome richness and ecology. Gut. 2016:65:426-36.

51. Dao MC, Belda E, Prifti E, Everard A, Kayser BD, Bouillot J-L, et al. Akkermansia muciniphila abundance is lower in severe obesity, but its increased level after bariatric surgery is not associated with metabolic health improvement. Am J Physiol Endocrinol Metab. 2019;317:E446-59.

52. Zhang $X$, Zhong H, Li Y, Shi Z, Ren H, Zhang Z, et al. Sex- and age-related trajectories of the adult human gut microbiota shared across populations of different ethnicities [Internet]. Nat Aging. 2021:87-100 Available from: https:// doi.org/10.1038/s43587-020-00014-2.

53. Zhou K. Strategies to promote abundance of Akkermansia muciniphila, an emerging probiotics in the gut, evidence from dietary intervention studies. J Funct Foods. 2017;33:194-201.

54. Alcock BP, Raphenya AR, Lau TTY, Tsang KK, Bouchard M, Edalatmand A, et al. CARD 2020: antibiotic resistome surveillance with the comprehensive antibiotic resistance database. Nucleic Acids Res. 2020;48:D517-25.

55. Druart C, Plovier H, Van Hul M, Brient A, Phipps KR, de Vos WM, et al. Toxicological safety evaluation of pasteurized Akkermansia muciniphila. J Appl Toxicol. 2021;41:276-90.

56. Degnan PH, Taga ME, Goodman AL. Vitamin B12 as a modulator of gut microbial ecology. Cell Metab. 2014;20:769-78.

57. Ottman N, Davids M, Suarez-Diez M, Boeren S, Schaap PJ, Martins Dos Santos VAP, et al. Genome-scale model and omics analysis of metabolic capacities of Akkermansia muciniphila reveal a preferential mucin-degrading lifestyle. Appl Environ Microbiol. 2017;83 Available from: https://doi.org/10.1128/AEM.01014-17.

58. Mojica FJM, Rodriguez-Valera F. The discovery of CRISPR in archaea and bacteria. FEBS J. 2016;283:3162-9.

59. Hamilton TA, Pellegrino GM, Therrien JA, Ham DT, Bartlett PC, Karas BJ, et al. Efficient inter-species conjugative transfer of a CRISPR nuclease for targeted bacterial killing. Nat Commun. 2019;10:4544.

60. Wirbel J, Pyl PT, Kartal E, Zych K, Kashani A, Milanese A, et al. Meta-analysis of fecal metagenomes reveals global microbial signatures that are specific for colorectal cancer. Nat Med. 2019;25:679-89.

61. Makarova KS, Wolf YI, Iranzo J, Shmakov SA, Alkhnbashi OS, Brouns SJJ, et al. Evolutionary classification of CRISPR-Cas systems: a burst of class 2 and derived variants. Nat Rev Microbiol. 2020;18:67-83. 
62. Russel J, Pinilla-Redondo R, Mayo-Muñoz D, Shah SA, Sørensen SJ. CRISPRCasTyper: an automated tool for the identification, annotation and classification of CRISPR-Cas loci [Internet]. Cold Spring Harbor Lab. 2020:2020.05.15.097824 [cited 2020 Dec 4]. Available from: https://www.biorxiv.org/content/10.1101/2020.05.15.097824v1.

63. Brister JR, Ako-Adjei D, Bao Y, Blinkova O. NCBI viral genomes resource. Nucleic Acids Res. 2015;43:D571-7.

64. Leenay RT, Maksimchuk KR, Slotkowski RA, Agrawal RN, Gomaa AA, Briner AE, et al. Identifying and visualizing functional PAM diversity across CRISPR-Cas systems. Mol Cell. 2016;62:137-47.

65. Tytgat HLP, Douillard FP, Reunanen J, Rasinkangas P, Hendrickx APA, Laine PK, et al. Lactobacillus rhamnosus GG outcompetes Enterococcus faecium via mucus-binding pili: evidence for a novel and heterospecific probiotic mechanism. Appl Environ Microbiol. 2016;82:5756-62.

66. Manara S, Asnicar F, Beghini F, Bazzani D, Cumbo F, Zolfo M, et al. Microbial genomes from non-human primate gut metagenomes expand the primate-associated bacterial tree of life with over 1000 novel species. Genome Biol. 2019;20: 299.

67. Pasolli E, Schiffer L, Manghi P, Renson A, Obenchain V, Truong DT, et al. Accessible, curated metagenomic data through ExperimentHub. Nat Methods. 2017;14:1023-4.

68. LV QB, Li SH, Zhang Y, Wang YC, Peng YZ, Zhang XX. A thousand metagenome-assembled genomes of Akkermansia reveal new phylogroups and geographical and functional variations in human gut. bioRxiv [Internet]. 2020; biorxiv.org. Available from: https://www.biorxiv.org/content/10.1101/2020.09.10.292292v1.abstract.

69. Shetty SA, Zuffa S, Bui TPN, Aalvink S, Smidt H, De Vos WM. Reclassification of Eubacterium hallii as Anaerobutyricum hallii gen. nov., comb. nov., and description of Anaerobutyricum soehngenii sp. nov., a butyrate and propionateproducing bacterium from infant faeces. Int J Syst Evol Microbiol. 2018;68:3741-6.

70. Belzer C, Chia LW, Aalvink S, Chamlagain B, Piironen V, Knol J, et al. Microbial metabolic networks at the mucus layer lead to diet-independent butyrate and vitamin B12 production by intestinal symbionts. MBio. 2017;8 Available from: https://doi.org/10.1128/mBio.00770-17.

71. Routy B, Le Chatelier E, Derosa L, Duong CPM, Alou MT, Daillère R, et al. Gut microbiome influences efficacy of PD-1based immunotherapy against epithelial tumors. Science. 2018;359:91-7.

72. Matson V, Fessler J, Bao R, Chongsuwat T, Zha Y, Alegre M-L, et al. The commensal microbiome is associated with antiPD-1 efficacy in metastatic melanoma patients. Science. American Association for the Advancement of Science. 2018; 359:104-8.

73. Nurk S, Meleshko D, Korobeynikov A, Pevzner PA. metaSPAdes: a new versatile metagenomic assembler. Genome Res. 2017;27:824-34.

74. Li D, Luo R, Liu C-M, Leung C-M, Ting H-F, Sadakane K, et al. MEGAHIT v1.0: a fast and scalable metagenome assembler driven by advanced methodologies and community practices. Methods. 2016;102:3-11.

75. Kang DD, Li F, Kirton E, Thomas A, Egan R, An H, et al. MetaBAT 2: an adaptive binning algorithm for robust and efficient genome reconstruction from metagenome assemblies. PeerJ. 2019;7:e7359.

76. Ondov BD, Treangen TJ, Melsted P, Mallonee AB, Bergman NH, Koren S, et al. Mash: fast genome and metagenome distance estimation using MinHash. Genome Biol. 2016;17:132.

77. Katoh K, Standley DM. MAFFT: iterative refinement and additional methods. Methods Mol Biol. 2014:1079:131-46.

78. Seemann T. Prokka: rapid prokaryotic genome annotation. Bioinformatics. 2014;30:2068-9.

79. Suzek BE, Wang Y, Huang H, McGarvey PB, Wu CH, UniProt Consortium. UniRef clusters: a comprehensive and scalable alternative for improving sequence similarity searches. Bioinformatics. 2015;31:926-32.

80. Buchfink B, Xie C, Huson DH. Fast and sensitive protein alignment using DIAMOND [Internet]. Nat Methods. 2015:59-60 Available from: https://doi.org/10.1038/nmeth.3176.

81. Steinegger M, Söding J. MMseqs2 enables sensitive protein sequence searching for the analysis of massive data sets. Nat Biotechnol. 2017;35:1026-8.

82. Mirdita M, von den Driesch L, Galiez C, Martin MJ, Söding J, Steinegger M. Uniclust databases of clustered and deeply annotated protein sequences and alignments. Nucleic Acids Res. 2017:45:D170-6.

83. McGinnis S, Madden TL. BLAST: at the core of a powerful and diverse set of sequence analysis tools. Nucleic Acids Res. 2004;32:W20-5.

84. Camacho C, Coulouris G, Avagyan V, Ma N, Papadopoulos J, Bealer K, et al. BLAST+: architecture and applications. BMC Bioinformatics. 2009:10:421.

85. Capella-Gutiérrez S, Silla-Martínez JM, Gabaldón T. trimAl: a tool for automated alignment trimming in large-scale phylogenetic analyses. Bioinformatics. 2009;25:1972-3.

86. Stamatakis A. RAXML version 8: a tool for phylogenetic analysis and post-analysis of large phylogenies. Bioinformatics. 2014;30:1312-3.

87. Truong DT, Franzosa EA, Tickle TL, Scholz M, Weingart G, Pasolli E, et al. MetaPhIAn2 for enhanced metagenomic taxonomic profiling. Nat Methods. 2015;12:902-3.

88. Longmead B, Salzberg SL. Fast gapped-read alignment with Bowtie2. Nat Methods. 2012; Available from: https:/www. sid.ir/en/journal/NiewPaper.aspx?1D=436196.

89. Computing RFS. R: a language and environment for statistical computing. Vienna: R Core Team; 2013. yumpu.com Available from: https:/www.yumpu.com/en/document/view/6853895/r-a-language-and-environment-for-statisticalcomputing

90. Kim S. ppcor: an R package for a fast calculation to semi-partial correlation coefficients. Commun Stat Appl Methods. 2015;22:665-74.

91. Balduzzi S, Rücker G, Schwarzer G. How to perform a meta-analysis with R: a practical tutorial. Evid Based Ment Health. Royal College of Psychiatrists. 2019;22:153-60.

92. Seabold S, Perktold J. Statsmodels: econometric and statistical modeling with Python [Internet]. Proceedings of the 9th Python in Science Conference. 2010. Available from: https://doi.org/10.25080/majora-92bf1922-011

93. Shelton AN, Seth EC, Mok KC, Han AW, Jackson SN, Haft DR, et al. Uneven distribution of cobamide biosynthesis and dependence in bacteria predicted by comparative genomics. ISME J. 2019;13:789-804.

94. Aramaki T, Blanc-Mathieu R, Endo H, Ohkubo K, Kanehisa M, Goto S, et al. KofamKOALA: KEGG Ortholog assignment based on profile HMM and adaptive score threshold. Bioinformatics. 2020;36:2251-2. 
95. van der Ark KCH, Aalvink S, Suarez-Diez M, Schaap PJ, de Vos WM, Belzer C. Model-driven design of a minimal medium for Akkermansia muciniphila confirms mucus adaptation. Microb Biotechnol. 2018;11(3):476-85.

96. Yin Y, Mao X, Yang J, Chen X, Mao F, Xu Y. dbCAN: a web resource for automated carbohydrate-active enzyme annotation. Nucleic Acids Res. 2012:40:W445-51.

97. Zhang H, Yohe T, Huang L, Entwistle S, Wu P, Yang Z, et al. dbCAN2: a meta server for automated carbohydrate-active enzyme annotation. Nucleic Acids Res. 2018;46:W95-101.

98. Cantarel B, Coutinho P, Henrissat B. Carbohydrate-active enzymes database, metagenomic expert resource [Internet]. Encyclopedia Metagenomics. 2012:1-7 Available from: https://doi.org/10.1007/978-1-4614-6418-1_25-10.

99. Leinonen R, Sugawara H, Shumway M. International Nucleotide Sequence Database Collaboration. The sequence read archive. Nucleic Acids Res. 2011;39:D19-21.

100. Minot S, Bryson A, Chehoud C, Wu GD, Lewis JD, Bushman FD. Rapid evolution of the human gut virome. Proc Natl Acad Sci U S A. 2013;110:12450-5.

101. Ly M, Jones MB, Abeles SR, Santiago-Rodriguez TM, Gao J, Chan IC, et al. Transmission of viruses via our microbiomes. Microbiome. 2016;4:64.

102. Norman JM, Handley SA, Baldridge MT, Droit L, Liu CY, Keller BC, et al. Disease-specific alterations in the enteric virome in inflammatory bowel disease. Cell. 2015;160:447-60.

103. Hannigan GD, Duhaime MB, Ruffin MT 4th, Koumpouras CC, Schloss PD. Diagnostic potential and interactive dynamics of the colorectal cancer virome. MBio. 2018;9 Available from: https://doi.org/10.1128/mBio.02248-18.

104. Liang G, Conrad MA, Kelsen JR, Kessler LR, Breton J, Albenberg LG, et al. The dynamics of the stool virome in very early onset inflammatory bowel disease. J Crohns Colitis. 2020; Available from: https://doi.org/10.1093/ecco-jcc/jjaa094.

105. Krueger F. Trim galore. A wrapper tool around Cutadapt and FastQC to consistently apply quality and adapter trimming to FastQ files. 2015;516:517. https://github.com/FelixKrueger/TrimGalore.

106. Zolfo M, Pinto F, Asnicar F, Manghi P, Tett A, Bushman FD, et al. Detecting contamination in viromes using ViromeQC Nat Biotechnol. 2019;37:1408-12.

107. Altschul SF, Gish W, Miller W, Myers EW, Lipman DJ. Basic local alignment search tool. J Mol Biol. 1990;215:403-10.

108. Rognes T, Flouri T, Nichols B, Quince C, Mahé F. VSEARCH: a versatile open source tool for metagenomics. PeerJ. 2016:4: e2584.

109. Zulkower V, Rosser S. DNA Features Viewer: a sequence annotation formatting and plotting library for Python Bioinformatics. 2020;36:4350-2.

110. Tareen A, Kinney JB. Logomaker: beautiful sequence logos in Python. Bioinformatics. 2020:36:2272-4.

111. Quinlan AR, Hall IM. BEDTools: a flexible suite of utilities for comparing genomic features. Bioinformatics. 2010;26:841-2.

112. Karcher N, Nigro E, Punčochář N, Blanco-Míguez A, Ciciani M, Manghi P, et al. Supplementary Data for Genomic diversity and ecology of human-associated Akkermansia species in the gut microbiome revealed by extensive metagenomic assembly. Zenodo. 2021. https://doi.org/10.5281/zenodo.5018705.

\section{Publisher's Note}

Springer Nature remains neutral with regard to jurisdictional claims in published maps and institutional affiliations.

Ready to submit your research? Choose BMC and benefit from:

- fast, convenient online submission

- thorough peer review by experienced researchers in your field

- rapid publication on acceptance

- support for research data, including large and complex data types

- gold Open Access which fosters wider collaboration and increased citations

- maximum visibility for your research: over $100 \mathrm{M}$ website views per year

At $B M C$, research is always in progress.

Learn more biomedcentral.com/submissions 